\title{
Evaluation of Bayesian Model and MCMC Validity in Verification of Piecewise Smooth Signature
}

(Penilaian Model Bayesian dan Kesahihan MCMC dalam Mengesahkan

Kelicinan Tandatangan Cebis demi Cebis)

\author{
M. BEHBOUd**, E. PASHA \& K. SHAFIE
}

\begin{abstract}
McKeague offered a new method for verification of off-line signature based on Bayesian Model and Markov Chain Monte Carlo (MCMC), in which smoothness of the signature curve seems a necessity (it should have no singular point), but when a signature is piecewise smooth, can we use this method for verification of this signature? If yes, how can we use that? And is this method appropriate for piecewise-smooth signatures too? In the current article, we give an idea for verification of a piecewise smooth signature based on McKeague's method. We suggest to separate the smooth segments from singular points and then each segment is verified by McKeague's method independently. Finally, according to the result from smooth segments, we determine the correctness of this signature. Then we will check the validity of this idea with computing errors via simulation.
\end{abstract}

Keywords: Biometric identification; functional data analysis; spatial point process; time warping

ABSTRAK

McKeague menawarkan satu kaedah baru untuk pengesahan tandatangan luar talian berdasarkan Model Bayesian dan Rantai Markov Monte Carlo (MCMC) dengan kelancaran lengkung tandatangan seolah-olah satu keperluan (ia tidak seharusnya mempunyai titik tunggal), tetapi apabila kelicinan tandatangan cebis demi cebis, bolehkah kita gunakan kaedah ini untuk pengesahan tandatangan ini? Jika ya, bagaimana boleh kita menggunakannya? Adakah kaedah ini juga sesuai untuk kelicinan tandatangan cebis demi cebis? Dalam kajian ini, kami memberikan idea untuk pengesahan kelicinan tandatangan cebis demi cebis berdasarkan kaedah McKeague. Kami cadangkan supaya segmen kelicinan dipisahkan daripada titik tunggal dan setiap segmen kemudiannya disahkan melalui kaedah McKeague secara berasingan. Kesimpulannya, berdasarkan hasil daripada segmen kelicinan, kami menentukan ketepatan tandatangan ini. Kemudian kami akan menyemak kesahihan idea ini dengan kesilapan pengkomputeran melalui simulasi.

Kata kunci: Analisis data fungsi; masa meleding; pengenalan biometrik; proses titik reruang

\section{INTRODUCTION}

Every day, millions of signatures are being produced, most of them applied to biometric identification. There are also a few signatures used for evaluation of checks, deals, official documents and business contracts. Though many studies have been carried out in the fields of signature verification and we have observed many salient developments in this regard, verification of signature has been regarded as an important and challenging subject.

Finding an appropriate statistical model to examine a signature is one of the well sought after researcher favorites. The systems of on-line signature verification have greatly developed (Plamondon. 2000). Hastie et al (1991) introduced a method based on dynamic time warping (DTW) and area of geometric shape analysis for verification of on-line signature. Ramsay $(2000,1997)$ have applied functional data analysis for verification of on-line signature. System of serial three stage multi-expert is presented to solve signature verification problems in Sansone and Vento (2000).
Although in recent years a number of studies have been carried out regarding off-line signature, the problem of verification of off-line signature has experienced no outstanding achievements compared with the on-line method. The reason might be the absence of information about velocity, pressure and hand angle. Kalera et al. (2003) describe an exquisite approach for off-line signature verification and identification using gradient, structural and concavity (GSC) features for feature extraction. Several learning strategies for signature verification were evaluated using a high-dimensional feature space that captures both local geometric information as well as stroke information by Srihari et al. (2004). Some methods for verification of off-line signature introduce a distance based nonparametric non-Bayesian method. These plans was to find changes and similarities (distances) among genuine signature samples and then using them for classification of the new signature sample. By this, forgery or correctness can be determined. As an example, please refer to Srihari et al. (2008). Fang et al (2003) and Matsuura and Sakai 
(1996) have proposed a number of other important methods regarding off-line signature verification.

A method for verification of off-line signature based on Bayesian model and MCMC approach developed by McKeague (2005), and with the aid of this method and using MATLAB software, successfully verified ' $S$ ' in Shakespeare's signature that has a smooth curve.

In this study, we give an idea for verification of piecewise-smooth signatures based on McKeague's method and we did by using $\mathrm{R}$ software. An Iranian signature sample which has a singular point is verified by using this idea. In order to evaluate the validity of our idea, the errors are computed for this signature sample via simulation. The suitability of R software is checked for verification of piecewise-smooth signatures based on our idea.

This article is organized in the following manner:

In the next section, we will introduce the Bayesian model for this research. Here, at first some basics of geometry of curves will be expressed and thereafter, the observation model, prior and posterior densities signature verification method will be discussed. In the section that follows, we will examine a signature sample through the method expressed. In the last section, we compute errors and discuss the presented idea.

\section{BAYESIAN MODEL}

In this section, we will introduce the proposed Bayesian model.

\section{BASIC DEFINITIONS}

It is essential at first to review some basics of geometry of curves. Consider parameterized curve $a:[a, b] \rightarrow \mathbb{R}^{2}$ with coordinate display $\alpha(t)=(x(t), y(t))^{\tau}$. We say that a curve is smooth (differentiable) whenever $x(t)$ and $y(t)$ at all points have derivatives of second order. In addition, we say the parameterized differentiable curve $a:[a, b] \rightarrow \mathbb{R}^{2}$ is regular if for each $t \in[a, b], \alpha^{\prime}(t) \neq 0$.

Supposing $t \in[a, b]$, the velocity vector (tangent) is in the form of:

$$
V(t)=\alpha^{\prime}(t)=\left(x^{\prime}(t), y^{\prime}(t)\right)^{\tau},
$$

In addition, arc-length is as follows:

$$
L(t)=\int_{a}^{t}\left|\alpha^{\prime}(u)\right| d u,
$$

where $\left|\alpha^{\prime}(u)\right|=\sqrt{\left(x^{\prime}(t)\right)^{2}+\left(y^{\prime}(t)\right)^{2}}$ is the length of the vector $\alpha^{\prime}(u)$.

The regular curve will be re-parameterized by arclength as, $\beta(s)=\alpha\left(L^{-1}(s)\right), s \in[0, L(b)]$. The curvature for curves parameterized by arc-length is defined as follows:

$$
\kappa(t)=x^{\prime}(t) y^{\prime \prime}(t)-x^{\prime \prime}(t) y^{\prime}(t) \text {. }
$$

The trace of curve is image set $([a, b])$. For more details refer to Carmo (1976).

One of the important features of curvature is that for $c$ $>0$, the curve $c a(t)$ has curvature $\frac{\kappa(t)}{c}$. When we compare a number of sample personal signatures, at first sight, it may seem that they have the same length; though, they are a little different. Therefore, we should standardize the observed signatures to be able to compare them. To do this, we rescale them to have equal arc-lengths $\tau>0$. As a result, the curvature of all signatures belongs to $C[0, \tau]$ (space of continuous function on $[0, \tau]$ ).

\section{OBSERVATION MODEL AND LOG-LIKELIHOOD}

In this section, we present the observation model and the log-likelihood. As noted before, since velocity, acceleration, pressure and hand angle are not available in off-line signatures, the signature is presented through its curvatures. Consequently, it becomes an essential that tangent (velocity vector) exists in all points of the curve. In addition, in the parameterized differentiable curve $a:[a, b]$ $\rightarrow \mathbb{R}^{2}$, the tangent exists if for each $t \in[a, b], \alpha^{\prime}(t) \neq 0$. As a result, there is no tangent in singular points. Therefore, this method is only possible for smooth signatures or smooth segments of the signature. Our plan for verification of signature which has singular points (which is piecewisesmooth) is to separate smooth segments of this signature from singular points and then verify each one individually and finally evaluate the correctness of the signature based on obtained results.

At first, we choose one of the signature samples as a template. We denote throughout the paper, subscript $j=1$, $\ldots, m$, refers to segment $j$ in a signature.

Smooth and arc-length parameterized approximation for signature curve, which is attained by image processing is named $\alpha_{j, o b s}(t), t \in\left[0, \tau_{j}\right]$. Then we calculate the velocity vector $v_{j}(t)=\alpha_{j, o b s}^{\prime}(t), t \in\left[0, \tau_{j}\right]$ and also the arc-length and curve curvature of the signature, using (2) and (3).

The observed velocity vector is modeled as follows:

$$
\begin{aligned}
& V_{j}(t)=\left(\begin{array}{c}
\cos \theta_{j}(t) \\
\sin \theta_{j}(t)
\end{array}\right), \\
& \theta_{j}(t)=\varphi_{j}+\int_{0}^{t} \kappa_{j}(s) d s+\sigma_{j} W_{j}(t),
\end{aligned}
$$

where $\kappa_{j}=C\left[0, \tau_{j}\right]$, is the underlying curvature function, $W_{j}(t)$ is the standard Brownian motion and $\varphi_{j}$ is the angle of $V_{j}(0)$ with $\mathrm{x}$-axis. Here, $\theta_{j}(t)$ shows the angle of the velocity vector with $\mathrm{X}$-axis in a state that white noise with variance $\sigma_{j}^{2}>0$ is added to underlying curvature. Also, with $\alpha_{j}(t)=\alpha_{j}(0)+\int_{0}^{t} V_{j}(t) d t$ formula, we can construct the curve from the velocity vector.

Suppose that $\sigma_{j}$ is given, then the log-likelihood for is as follows (McKeague 2005):

$$
\ell\left(\kappa_{j} \mid V_{j}\right)=\frac{1}{\sigma_{j}^{2}} \int_{0}^{\tau_{j}} \kappa_{j}(s)\left\{V_{j, 1}(s) d V_{j, 2}(s)-d V_{j, 1}(s)\right\}-
$$




$$
\frac{1}{2 \sigma_{j}^{2}} \int_{0}^{\tau_{j}} \kappa_{j}(s)^{2} d s+\frac{1}{8} \sigma_{j}^{2} \tau_{j}
$$

To calculate the numerical evaluation of likelihood, velocity vector $V_{j}(t), t \in\left[0, \tau_{j}\right]$ are approximated on a regular grid $s_{n_{j}}=\frac{n_{j} \tau_{j}}{N_{j}}, n_{j}=0, \ldots, N_{j}$. Also in this article, the amount of $\sigma_{j}$ was chosen via experimental studies.

Now, we expand the model to signature samples. Because the obtained arc-lengths are different, we rescale all signatures in order acquire their arc-length equal to the template arc-length, . Then, the velocity vector for signature samples are computed. Suppose $V_{j}^{(i)}, i=1, \ldots, n$, is the velocity vector for $\mathrm{i}^{\text {th }}$ sample.

Some slight differences in velocity vectors of samples lead to the time difference in curvature peaks of samples. Accordingly, to make the model flexible, we use the time warp function $h_{j, i}:\left[0, \tau_{j}\right] \rightarrow\left[0, \tau_{j}\right], \mathrm{i}=1, \ldots, n$, as hidden variables or random effects.

The full log-likelihood for vector of time-warp functions $\boldsymbol{h}_{j}=\left(h_{j, i} ; i=1, \ldots, n\right)$ and underlying curvature function is as below:

$$
\ell\left(\kappa_{j}, \boldsymbol{h}_{j} \mid \text { data }_{j}\right)=\sum_{i=1}^{n} \ell\left(\kappa_{j, i} \mid V_{j}^{(i)}\right),
$$

where $\kappa_{j, i}=\kappa_{j}\left(h_{j, i}\right)$ is the curvature function segment $j$ of sample $i$.

\section{PRIOR}

As noted before, $V_{j}(i), i=1, \ldots, n$, are model observations, and $\boldsymbol{h}_{j}$ and $\boldsymbol{\kappa}_{j}$ are the parameters. According to the Bayesian model definitions, we define an appropriate prior distribution for the parameters named as baseline curvature process and time warping processes.

Baseline curvature process We choose the curvature of the template sample as a template curvature function and name it $\kappa_{j, 0}$. Then by specifying an appropriate value for $\varepsilon_{j}$, we define the buffer region around the $\kappa_{j, 0}$ as:

$$
\mathfrak{B}_{j}=\left\{(t, y): \kappa_{j, L}(t) \leq y \leq \kappa_{j, U}(t), t \in\left[0, \tau_{j}\right]\right\},
$$

where $\kappa_{j, U}(t)=K_{j, 0}(t)+\varepsilon_{j}$ and $\kappa_{j, L}(t)=\kappa_{j, 0}(t)-\varepsilon_{j}$ are template curvature bounds. Supposing that $X_{j}$, is the baseline curvature process of segment $j$ and to be a Strauss process i.e. it has unnormalized density:

$$
f\left(\boldsymbol{x}_{j}\right)=\beta_{j}^{n\left(x_{j}\right)} \gamma_{j}^{d\left(x_{j}\right)}
$$

with respect to unite rate Poisson process in $\mathfrak{B}_{j}$, the points $\left(t_{j, m_{j}}, y_{j, m_{j}}\right), m_{j}=1, \ldots, n\left(X_{j}\right)$, that $0<t_{j, 1}<t_{j, 2}<\ldots<t_{j, n\left(X_{j}\right)}<\tau_{j}$ will be generated completely randomly in the buffer region $\mathfrak{B}_{j}$ where $0 \leq \gamma_{j} \leq 1$ and $\beta_{j}>0$ are tuning parameters, $d\left(\boldsymbol{x}_{j}\right)$ is the of paired points that their maximum metric distance (distance between their time coordinate) is $\rho_{j}$ and $n\left(\boldsymbol{x}_{j}\right)$ is the total number of randomly generated points. See van
Lieshout (2000) for details. By adding $\left(t_{j, n(X)+1}, y_{j, n(X)+1}\right)=$ $\left(\tau_{j}, \kappa_{j, 0}\left(\tau_{j}\right)\right)$ and $\left(t_{j, 0}, y_{j, 0}\right)=\left(0, \kappa_{j, 0}(0)\right)$ as the fixed member to randomly generated points, we define baseline curvature function as bellow (McKeague 2005):

$$
\begin{aligned}
\kappa_{j}(t)= & \left(\frac{t_{j, m_{j+1}}-t}{t_{j, m_{j+1}}-t_{j, m_{j}}}\right)\left\{\kappa_{j, 0}(t)+y_{j, m_{j}}-\kappa_{j, 0}\left(t_{j, m_{j}}\right)\right\} \\
& +\left(\frac{t-t_{j, m_{j}}}{t_{j, m_{j+1}}-t_{j, m_{j}}}\right)\left\{\kappa_{j, 0}(t)+y_{j, m_{j+1}}-\kappa_{j, 0}\left(t_{j, m_{j+1}}\right)\right\},
\end{aligned}
$$

where $t_{j, m_{j}} \leq t \leq t_{j, m_{j+1}}, m_{j}=0,1, \ldots, n\left(X_{j}\right)$.

Time warping process The time warping process, is an increasing and continuous process, which is linear between the grid points $u_{j, s}=\frac{s_{j} \tau_{j}}{p_{j}}, s_{j}=0, \ldots, p_{j}$ and constrained so that $h_{j, i}\left(\tau_{j}\right) \leq \tau_{j}$. Also is determined by the user. The parameters $\theta_{j, s_{j}}=h_{j, i}\left(u_{j, s_{j}}\right)-h_{j, i}\left(u_{j, s_{j}}\right), s_{j}=1, \ldots, p_{j}$, determine $h_{j, i}$ and we combine $\theta_{j, i s_{j}}$ into a vector $\boldsymbol{\theta}_{j} \in 0_{j}=\left[0, \tau_{j}\right]^{n p_{j}}$ to give $\boldsymbol{h}_{j}$.

To provide the prior on $h_{j, i}$, we utilize functional data analysis in curve registration. Prior density on $h_{j, i}$ (base on its parameters) is specified as follows:

$$
\pi_{j}\left(h_{j, i}\right) \propto \exp \left\{-\eta_{j} J_{j}\left(h_{j, i} \mid \kappa_{j}\right)\right\}
$$

where $\eta_{j}>0$ is the precision parameter determined by the user. In this method, deviation of data from the fitted velocity vector is penalized and its function is as follows:

$$
J_{j}\left(h_{j, i} \mid \kappa_{j}\right)=\int_{0}^{\tau_{j}} \operatorname{angle}\left(V_{j}^{(i)}(t), V_{j, \text { fitted }}^{(i)}(t)\right) d t .
$$

The velocity vector $V_{j, \text { fitted }}^{(i)}(t)$, is a velocity vector according to curvature function $\kappa_{j}\left(h_{j i}(t)\right)$ with an initial value of $V_{j}^{(i)}(0)=V_{j, \text { fitted }}^{(i)}(t)$ and $\operatorname{angle}(u, v)=\cos ^{-1}\left(u^{T} v\right)$ is the angle between unit vectors $u$ and $v$ in radians.

\section{POSTERIOR DISTRIBUTION AND SAMPLING FROM IT}

Assuming the base curvature $\kappa_{j}$ is shown with its $\boldsymbol{x}_{j}$ points, time warp vector $\boldsymbol{h}_{j}$ is shown with $\boldsymbol{\theta}_{j}$ and data are known. The posterior density is proportional to:

$$
\begin{aligned}
g\left(\boldsymbol{x}_{j}, \boldsymbol{\theta}_{j}\right)= & \exp \left\{\ell\left(\kappa_{j}, \boldsymbol{h}_{j} \mid \text { data }_{j}\right)\right\} \\
& f\left(\boldsymbol{x}_{j}\right) \prod_{i=1}^{n} \pi\left(h_{j, i} \mid \kappa_{j}, \text { data }_{j}\right),
\end{aligned}
$$

where the dominating measure is the product of unit-rate Poisson distribution on $\mathfrak{B}_{j}$ and the Lebesgue measure on $\Theta_{j}$.

The MCMC scheme used to detect this posterior is Metropolis within Gibbs. For background, refer to NtZoufras (2009). Each component $\theta_{j}$ of $\boldsymbol{\theta}_{j}$ is updated via the Random Walk Metropolis. The distribution of proposal $\theta_{j}^{\prime}$ is normal with zero mean and restricted to the compact interval $\left[0, \tau_{j}\right]$. The $\boldsymbol{x}_{j}$-component is updated by a Metropolis Hastings Algorithm, which was presented 
by Geyer and Moller (1994). According to this algorithm, either a point $\xi$ is born uniform randomly in $\mathfrak{B}$, where in this state we have a movement as $\left(\boldsymbol{x}_{j}, \boldsymbol{\theta}_{j}\right) \rightarrow\left(\boldsymbol{x}_{j} \cup \xi, \boldsymbol{\theta}_{j}\right)$ and the Hastings ratio is as follows:

$$
\alpha_{j, b}\left(x_{j}, \boldsymbol{\theta}_{j}, \xi\right)=\frac{\left|\mathfrak{B}_{j}\right|}{n\left(\boldsymbol{x}_{j}\right)+1} \frac{g\left(\boldsymbol{x}_{j} \cup \xi, \boldsymbol{\theta}_{j}\right)}{g\left(\boldsymbol{x}_{j}, \boldsymbol{\theta}_{j}\right)},
$$

or a point $\xi$ is randomly selected from the points inside and is killed. In this state, we have a movement as $\left(\boldsymbol{x}_{i}, \boldsymbol{\theta}_{j}\right)$ $\rightarrow\left(\boldsymbol{x}_{j} \backslash \xi, \boldsymbol{\theta}_{j}\right)$ and the Hastings ratio is as follows:

$$
\alpha_{j, d}\left(\boldsymbol{x}_{j}, \boldsymbol{\theta}_{j}, \xi\right)=\frac{n\left(\boldsymbol{x}_{j}\right) g\left(\boldsymbol{x}_{j} \backslash \xi, \boldsymbol{\theta}_{j}\right)}{\left|\mathfrak{B}_{j}\right| g\left(\boldsymbol{x}_{j}, \boldsymbol{\theta}_{j}\right)} .
$$

Moreover, the probability of born or killed points are equal and the mentioned $\left|\mathfrak{B}_{j}\right|$ in $\alpha_{j, b}$ and $\alpha_{j, d}$ is the buffer region area.

\section{SIGNATURE VERIFICATION}

As apparent from the study's title, it became clearer that the present paper aimed to evaluate correctness of a new signature that is technically called verification of new signature sample. We are going to do this via Bayesian model and MCMC scheme. What has been said up to this point are preliminaries we need for verification of the new signature.

We re-scale the new signature which we want to know that this is genuine signature or a forgery. Then we compute its velocity vector and name it $V^{\text {new }}$. To evaluate the correctness of this signature, first the forgery index $F_{j}$ should be calculated. Firstly, compare $V_{j}^{\text {new }}$ with each $V_{j}^{(i)}$, $i=1, \ldots, n$, in order to find the difference rate. So rotate $V_{j}^{\text {new }}$ clockwise to have the initial direction $V_{j}^{(i)}(0), i=1, \ldots$, $n$ and name it $V_{j, i}^{\text {new }} i=1, \ldots, n$. Next, compute the forgery index $F_{j}$ as bellow (McKeague 2005):

$$
F_{j}=\frac{1}{\pi \tau_{j}} \min _{i=1, \ldots, n} \int_{0}^{\tau_{j}} \operatorname{angle}\left(V_{j, i}^{\text {new }}(t), V_{j}^{(i)}(t)\right) d t .
$$

In fact, the forgery index calculates the difference between the new signature and the most similar signature sample to it. To range $F$ between 0 and 1, we normalize it. We expect it to be small for genuine signatures because small magnitude of forgery index indicates that the new signature is similar to one of the samples.

Actually, the null hypothesis $\left(H_{0}\right)$ is that the new signature is genuine and is the test statistic. The reason that new signature is a forgery is determined by p-value $P\left(F_{j}^{*}>F_{j}\right)$. The velocity vector $V_{j}^{*}$ is the velocity vector of the fitted observation model that has forgery index $F_{j}{ }_{j}$. Replacing $V_{j}^{\text {new }}$ by $V_{j}^{*}$ in $F_{j}$ to compute $F_{j}^{*}$.

$\mathrm{P}$-value is determined by bootstrap simulation. After MCMC scheme, we have posterior mean of baseline curvature, $\hat{\kappa}_{j}$ and posterior mean of time warping functions $h_{j, i}$ that is $\hat{h}_{j, i}$, and can easily compute $\hat{\kappa}_{j}\left(\hat{h}_{j, I}().\right)$ which $I$ is randomly selected from $i=1, \ldots, n$.
Then replace $\hat{\kappa}_{j}\left(\hat{h}_{j, l}().\right)$ by $\kappa_{j}$ model (5) and simulate it 10000 times by using bootstrap method. Now we compute p-value.

Moreover, our aimed was to assess the new signature validity in significance level 0.05 . To do this, suppose two segments of the signature are independent. As a result, we should evaluate the correctness of each segment in significance level 0.0255. Moreover, to accept the new signature as a genuine signature, it required both segments to be genuine and if one segment is a forgery, the signature will be rejected.

\section{APPLICATION}

In order to examine the correctness of a new signature sample, it is obligatory to possess information about that individual's signature. Achieving this, in the first stage, four samples of his/her signatures, shown in Figure 1, was obtained.

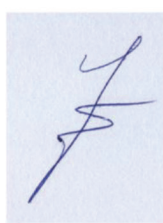

(1)

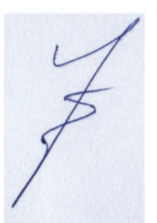

(2)

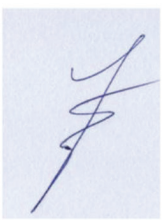

(3)

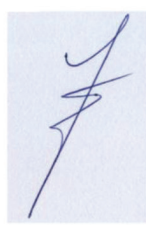

(4)
FIGURE 1. Four samples of the examined signature

Based on Figure 2, we separate smooth segments from singular points and consider each segment independently. Here, signature sample has one singular point. Whenever it has more than one singular point, the procedure is similar.

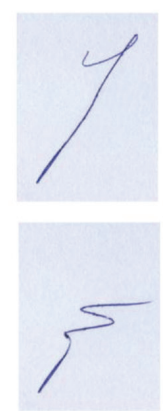

(1)

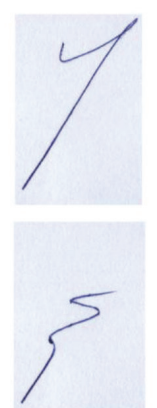

(2)

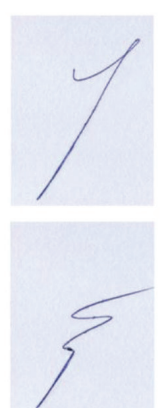

(3)

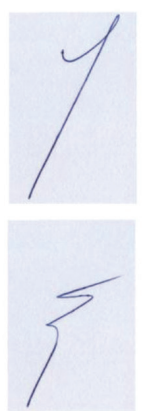

(4)
FIGURE 2. Separated segments of four sample signatures

Firstly, by use of the read.jpeg command we load the scanned image of the signature into software R. Secondly, we click on important points of the signature curve and register coordinates of these points. In the next stage, through interpolation by cubic spline functions in 1001 knots and separately in $\mathrm{x}$ and $\mathrm{y}$ coordinates, we calculate a 
smooth and relatively exact approximation for the signature curve. Now, selecting 100 knots from 1001 knots make a regular grid of 100 knots on the signature curve. The rule for choosing these knots is that knot 1 is the first knot (the first point we clicked on) and knot 1001 is the 100th knot (the point in which the signature arc-length is equal to, after re-scaling). The remaining 98 knots are located between these two knots and along the signature.

In this paper, we choose signature sample 3 as the template sample. Because these signatures have 2 smooth segments, subscript $j=1,2$ refer to segment in signatures.

We calculate $V_{j}(t), j=1,2$, by calculating component derivatives for 100 chosen knots. After calculating which was introduced in the previous section, arc-lengths of first and second segments, $\tau_{1}$ and $\tau_{2}$, were obtained 1.85320653861 and 2.180766843 , respectively. In this situation, 0.15 is an appropriate value for $\sigma_{j}, j=1,2$, in the model (4). Figure 3 shows the curve of both segments of the sample 3 , their simulated curvature, the third sample curvatures on which white noise superimposed and obtained trace from latter curvatures. Left part belongs to the first segment of the signature and the right part is related to the second one.

Now, we expend the model to 4 signature samples. At first we calculate the arc-length samples. The arclength of segment 1 for signature samples 1, 2 and 4 are $1.793639223,1.8657510341$ and 1.6417542252 , respectively, and the arc-length of segment 2 for signature samples 1,2, and 4 are 2.209253905, 1.909385592 and 1.907520967 , respectively. Because the arc-length of signature samples differs together, we re-scale signatures. Then, we calculated the $V_{1}^{(i)}$ and $V_{2}^{(i)}, \mathrm{i}=1,2,3,4$, which are demonstrated in Figures 4 and 5, respectively.
We choose the curvature function of signature sample 3 as the $\kappa_{j, 0}, j=1,2$ and here $\varepsilon_{1}=\varepsilon_{2}=0.5, \beta_{1}=5, \beta_{2}=2, \gamma_{1}$ $=\gamma_{2}=0.01, \rho_{1}=0.005 \tau_{1}$, and $\rho_{2}=0.001 \tau_{2}$ are appropriate values for the baseline curvature process.

As can be seen in Figure 6 the curvature function $\kappa_{j}(t), j=1,2$, is completely inside the buffer region and passes through points generated by the Strauss process. Furthermore, the resulted trace from the curvature function is compatible to shape of the signature.

In this study, $p_{1}=p_{2}=20$ and $\eta_{1}=\eta_{2}=500$ are suitable values for prior density parameters on $h_{j, i}$ and burn $=$ burn $=2000$ and afterburn $_{1}=$ afterburn $_{2}=2000$ are proper values for parameters related to doing MCMC scheme.

By running the MCMC program, an estimation of curvature function (posterior mean of curvature), and estimation for time-warping functions (posterior mean of time-warping functions $h, i=1,2,3,4$ ) was obtained.

Figure 7 shows the posterior mean of curvatures of segments 1 and 2 without posterior mean of time warping functions. As seen in the right images, the achieved traces (simulated curves) have a proper compatibility with the picture of observed signature samples.

It's worth noting $\varepsilon_{j}=2, j=1,2$, in Figures 6 and 7 . The acceptance rate for the used MCMC scheme for accept $\theta^{\prime}$, dead one point in $\boldsymbol{x}_{i}$ and born one point in $\boldsymbol{x}_{i}$ for first segment are 2,16.375 and $15.7 \%$ and for second segment are $1.275,14.625$ and $13.725 \%$, respectively.

It should mention that since the achieved results of MCMC appropriately adapt with the signature samples, the acceptance percentage becomes reasonable. The main reason for low acceptance rate to accept $\theta^{\prime}$ can be accepted and be found in the complexities of signature curves. Because of this, we should consider a very low acceptance
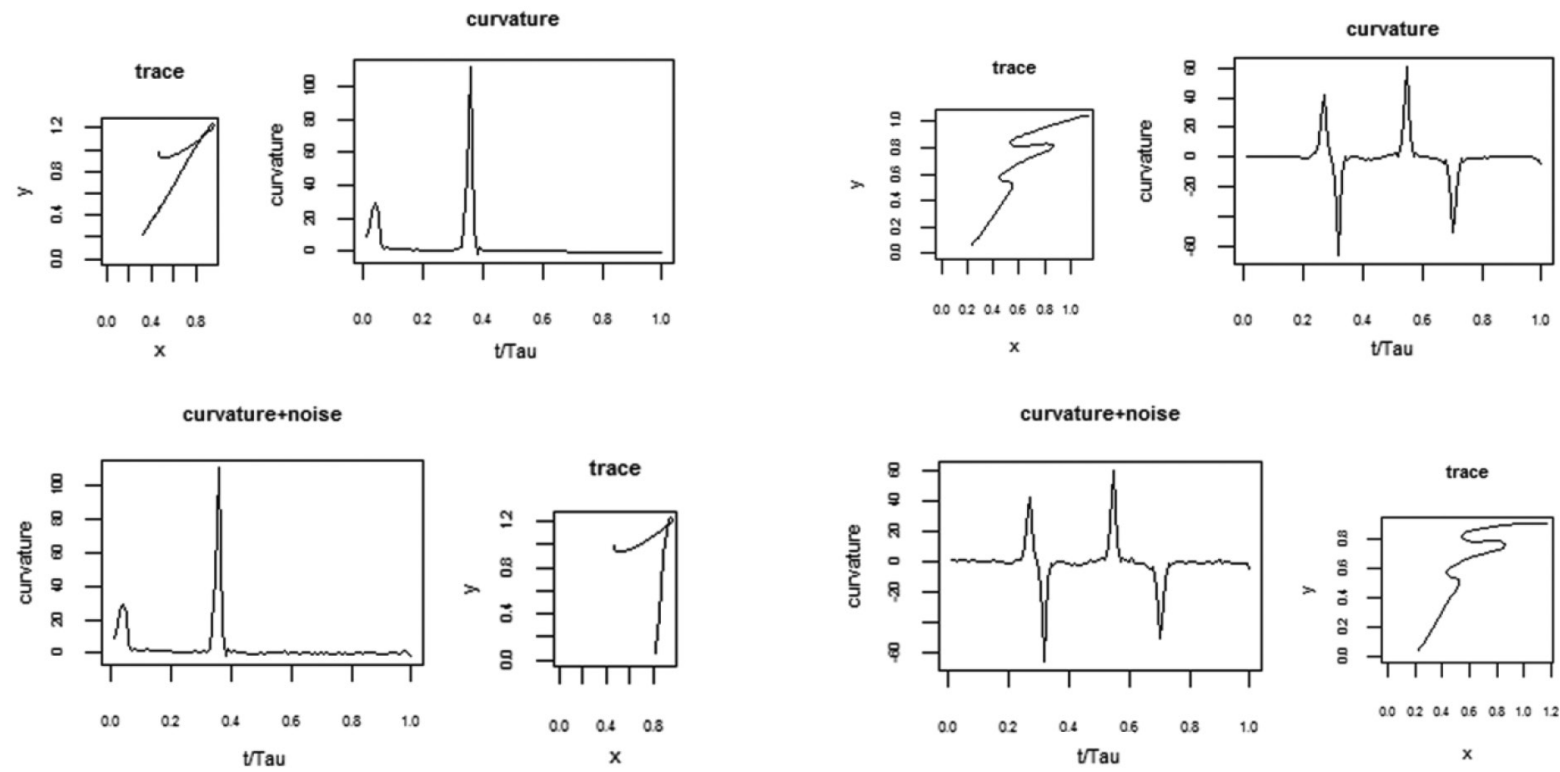

FIGURE 3. The curve of the segments of signature sample 3 (top-left row); simulated curvature from the segment curves of sample 3 (top-right row); curvatures of the third sample to which a little white noise is added (bottom-left row); re-constructed curves (obtained trace) from related curvatures (bottom-right row) 

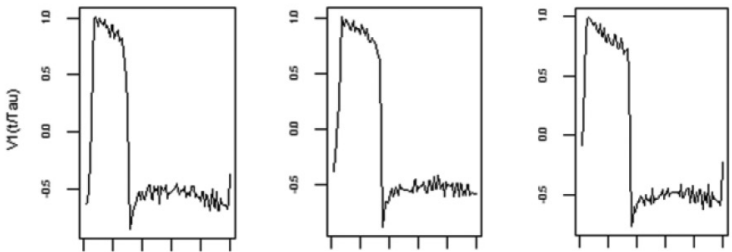

$\begin{array}{llllll}00 & 02 & 04 & 06 & 08 & 10\end{array}$

00 02 as ab as 10

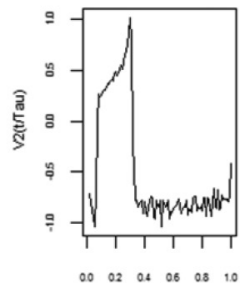

(1)

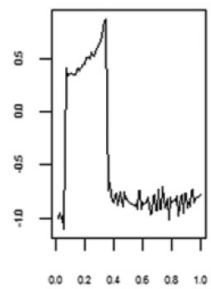

(2)
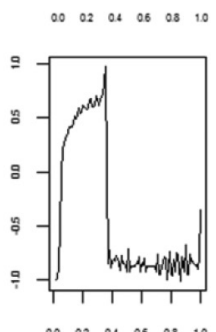

(3)

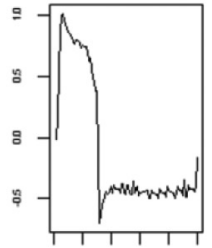

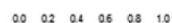

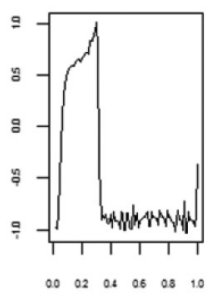

(4)

FIGURE 4. Components of velocity vector $V_{1}^{(i)}, i=1,2,3,4$

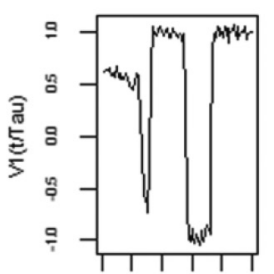

000204060810

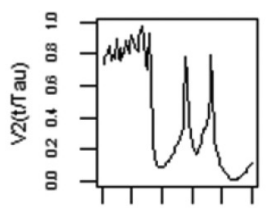

$0.0020 .40 .60 .8 \quad 1.0$

(1)

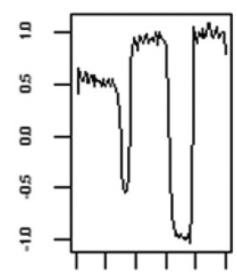

00020.4060810

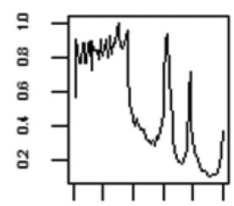

00020.40 .60 .810

(2)

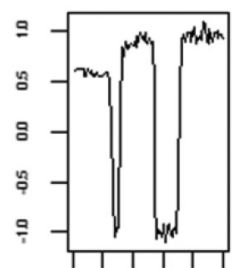

000204060810

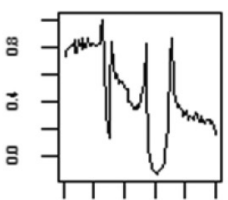

$0.0020 .40 .60 .8 \quad 1.0$

(3)

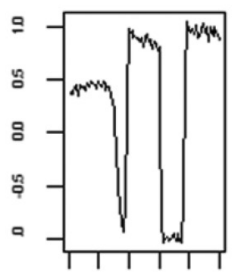

$0.0020 .40 .60 .8 \quad 1.0$

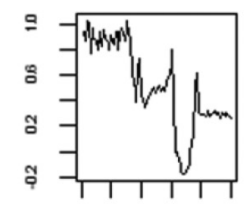

00020.4060 .81 .0

(4)

FIGURE 5. Components of velocity vector $V_{2}^{(i)}, i=1,2,3,4$
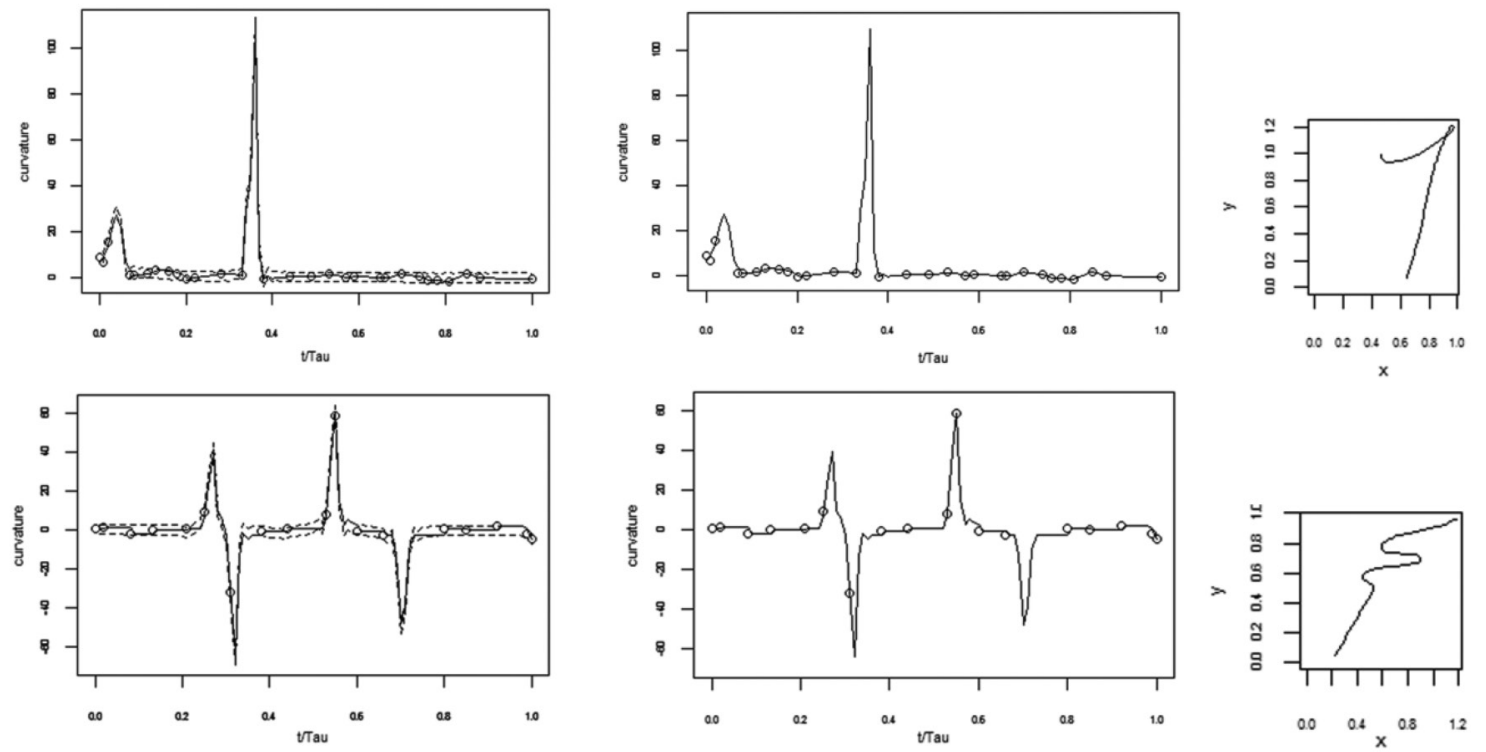

FIGURE 6. Bracketing functions for the buffer region (dotted line), generated points via Strauss process, and approximated curvature on points (bold line) (left); points generated via Strauss process and approximated curvature on points (bold line) (center); obtained trace from approximated curvature (right) 

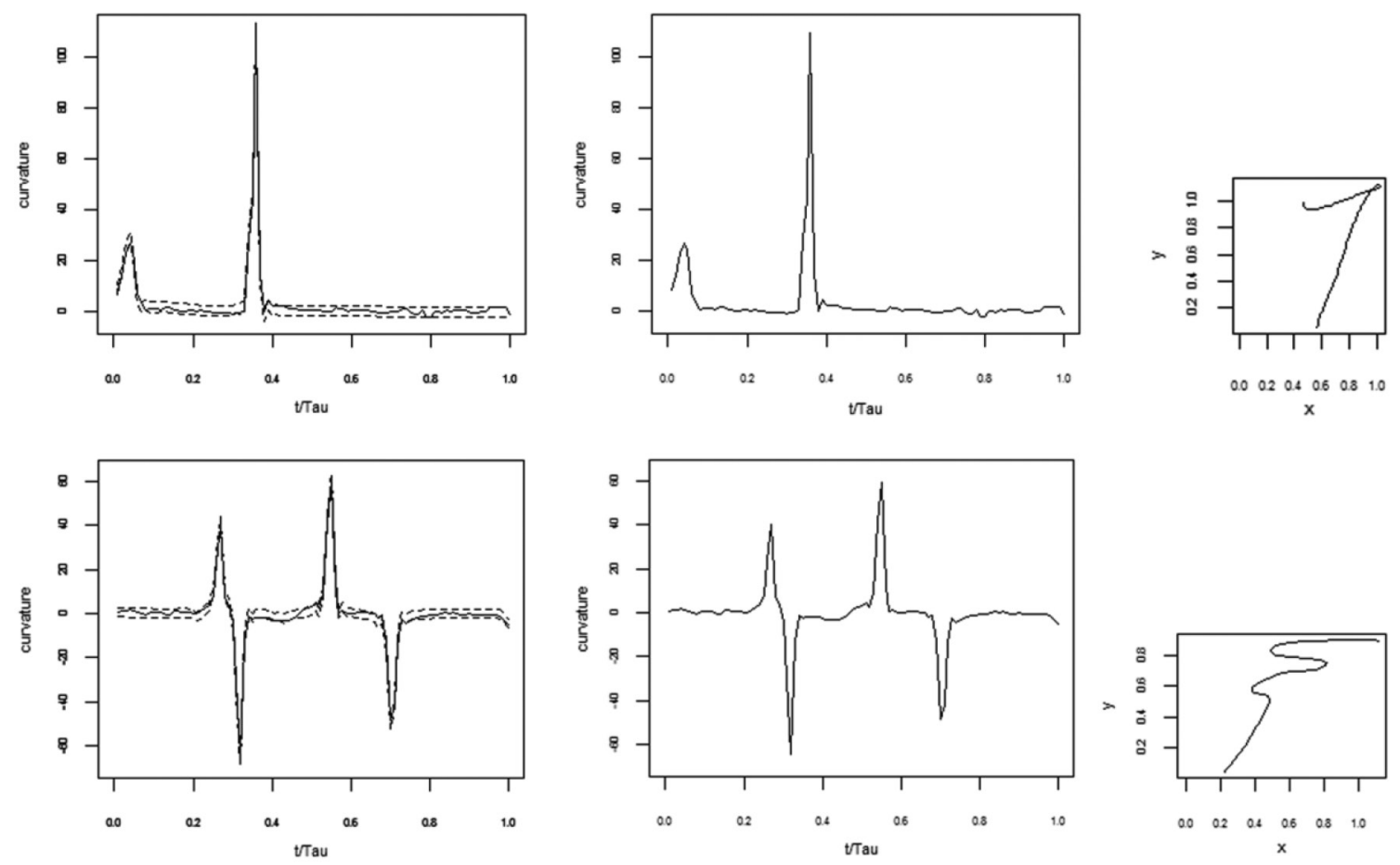

FIGURE 7. Posterior mean of curvature (bold line) and bracketing functions for the buffer region (dotted lines) (left); posterior mean of curvature (center); simulated curve (obtained trace) from the posterior mean of curvature (right)

rate. Figures 8 and 9 illustrate the reconstructed curves from the posterior mean of baseline curvature function and the posterior mean of time warping functions for the first and secong segments of samples, respectively.

To verify the correctness of a new signature, we scanned image of the new signature and separated its smooth segments from the singular point as presented in Figure 10 .
After the primary computations, the arc-length of segments 1 and 2 of the new signature are 1.8868456831 and 2.2399613739, respectively. We re-scale the new signature. Then we compute its velocity vector and name it $V^{\text {new }}$.

After calculating, as you can see in Figure 11, the forgery index of segments 1 and $2, F_{1}, F_{2}$, are 0.1158795 and 0.1721104 , respectively as well as the obtained
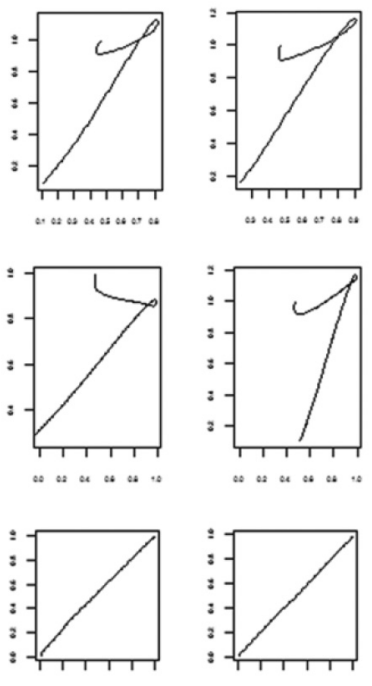

of of ac of at of
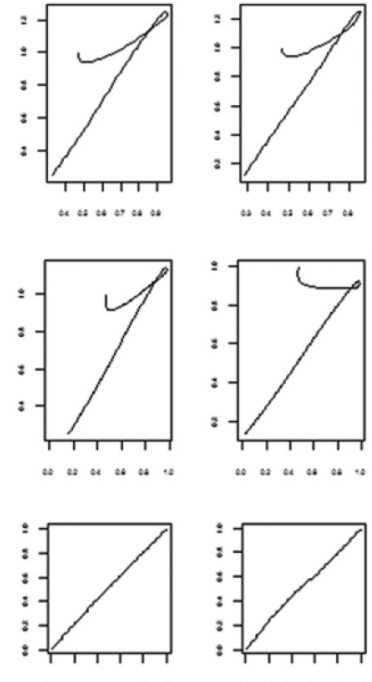

FIGURE 8. Traces of first segment of 4 signature samples(first line); reconstructed curves (obtained traces) from posterior mean of baseline curvature function that adjusted by posterior mean of time warping functions (the second line); posterior mean of time warping functions (the third line) 

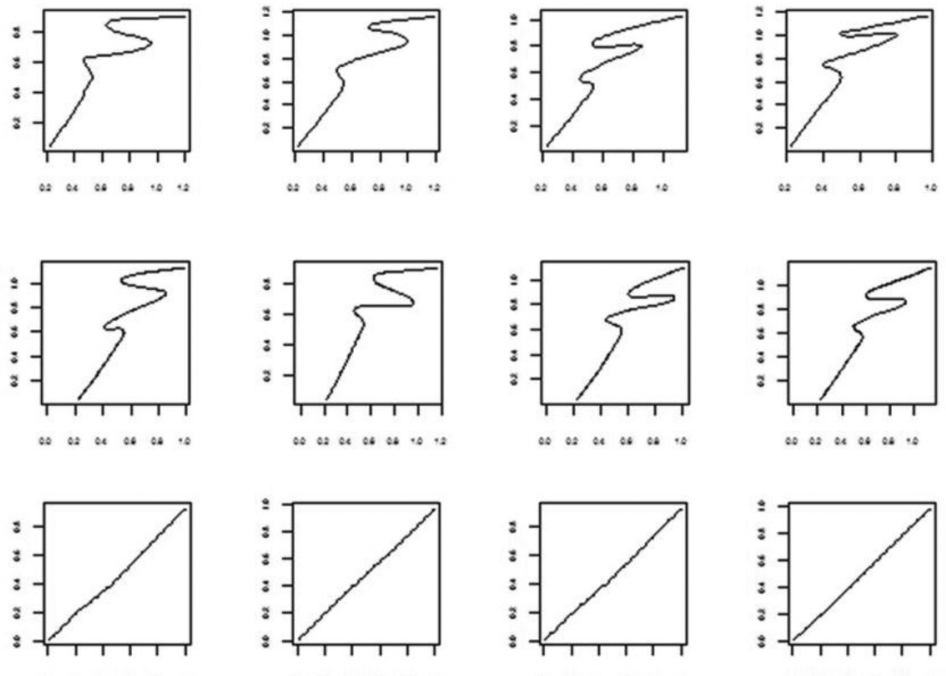

FIGURE 9. Traces of second segment of 4 signature samples (first line); reconstructed curves (obtained traces) from posterior mean of baseline curvature function that adjusted by posterior mean of time warping functions (the second line); posterior mean of time warping functions (the third line)
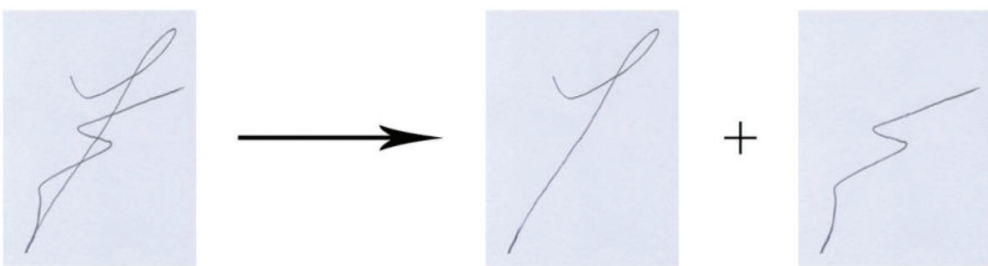

FIGURE 10. New signature; Smooth segments have separated from the singular point
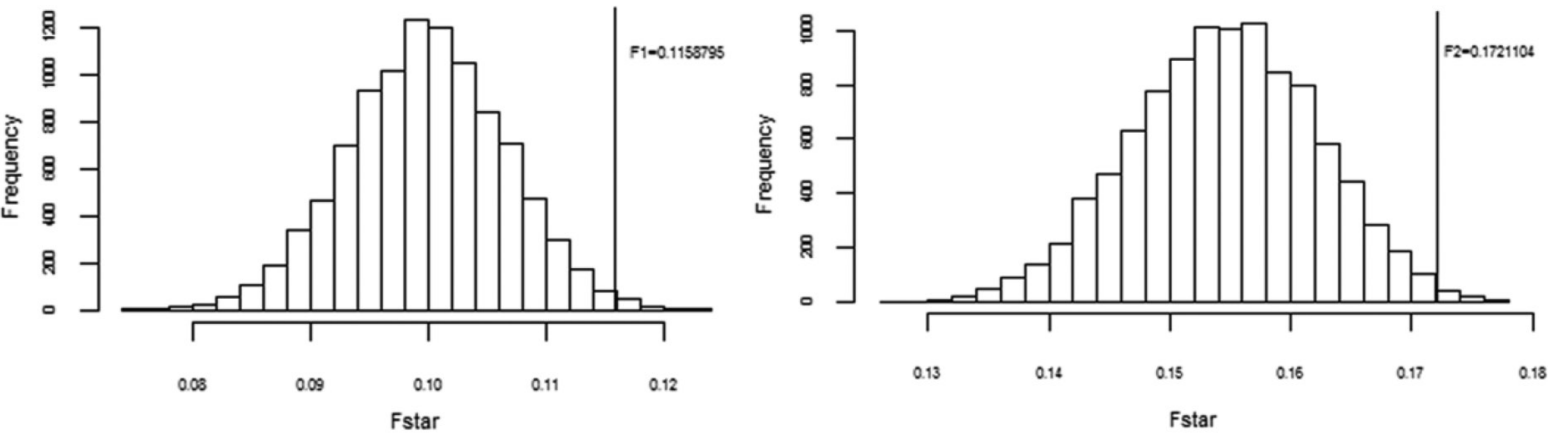

FIGURE 11. Simulated histogram of and vertical line as the forgery index for the first segment of the new signature (left); and simulated histogram of and vertical line as forgery index for segment 1 of new signature (right)

results for p-value of segments 1 and 2 are 0.006 and 0.0079 , respectively. Since the p-values are small, the null hypothesis in both segments is rejected. We conclude that the new signature is a forgery.

\section{DISCUSSION AND RESULTS}

In order to evaluate the validity of this method, we compute errors of this method. Therefore, we consider two signatures, one is a forgery and the other is a genuine signature. Then we examine to find the percentage of situations in which the genuine signature is identified as a forgery and find the percentage of situations in which the forgery is identified as a genuine signature. Figure 12 presents the scanned image of both forgery and genuine signature.

In order to compute errors, after the initial computations and re-scaling arc-length, we calculate the velocity vectors 

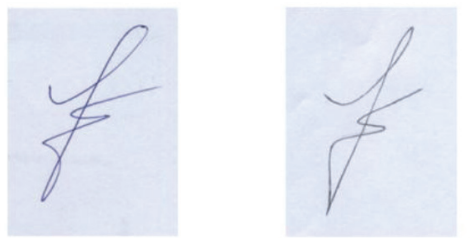

FIGURE 12. Genuine signature (left); Forgery (right)

of the genuine and forgery signatures. At the beginning, the percentage of situations in which the the genuine signature is defined as a forgery is calculated.

After calculation, the forgery index for segments 1 and 2 of the genuine signature, $F_{1}, F_{2}$, are obtained 0.0177887 and 0.01410058 , respectively. Then we simulate the index $F_{j}^{*}, j=1,2$, via bootstrap method and compute $P\left(F_{j}^{*}>\right.$ $\left.F_{j}\right), j=1,2$. In order to obtain error, we repeat this process 500 times and calculate the percentage of situations in which the genuine signature is identified as a forgery. Consequently, we can get the cases in which $P\left(F_{j}^{*}>F_{j}\right), j=$ 1,2 , is less than 0.0255 . Error of segments 1 and 2 are 0.01 and 0.012 , respectively. Suppose that signature segments are independent and considering that the signature is a forgery when at least one of the segments is a forgery, the error is equal to 0.02188 .

In order to compute the percentage of situations in which the forgery is defined as a genuine signature, after doing the initial computations, the forgery index for segments 1 and 2 of the forgery signature are 0.1261095 and 0.1541828 , respectively. As before, in order to obtain error, we compute $P\left(F_{j}^{*}>F_{j}\right), j=1,2$, in 500 times and find out the cases wherein $P\left(F_{j}^{*}>F_{j}\right), j=1,2$, was greater than 0.0255 . The error of segments 1 and 2 are 0.11 and 0.226 , respectively. Supposing the smooth segment is independent, the error will be 0.336 .

According to the attained results of errors, we calculate that the idea of separating smooth segments of the signature from singular points is a proper idea in verification of piecewise smooth signature by Bayesian model and MCMC scheme. Generally speaking, this could be a perfect method in verifying signatures of any type. It is necessary to note that through calibrating $\sigma_{j}, j=1,2$, we can optimize errors.

The required time for signature verification, in addition, will be less than 30 min with this method and using software R. Since it is free software and keeping in mind this fact that its applications can be provided as various packages for interested people, using this software would be better and more applicable.

To sum up, a question remains that do the Bayesian model and MCMC work well in verification of on-line signatures.

\section{ACKNOWLEDGEMENTS}

The authors would like to acknowledge the anonymous reviewers for their helpful comments on earlier draft of the manuscript.

\section{REFERENCES}

do. Carmo, M.P. 1976. Differential Geometry of Curves and Surfaces. New York: Prentice-Hall.

Fang, B., Leung, C.H., Tang, Y.Y., Tse, K.W., Kwok, P.C.K. \& Wong, Y.K. 2003. Off-line signature verification by the tracking of feature and stroke positions. Pattern Recognition 36: 91-101.

Geyer, C.J. \& Møller, J. 1994. Simulation and likelihood inference for spatial point processes. Scandinavian Journal of Statistics 21: 359-373.

Hastie, T.J., Kishon, E., Clark, M. \& Fan, J. 1991. A model for signature verification. Proceedings of the IEEE Conference on Systems. Man, and Cybernetics 1: 191-196.

Kalera, M.K., Zhang, B. \& Sriahri, S.N. 2003. Offline signature verification and identification using distance statistics. Proc. of the Int. Graphonomics society Conference. pp. 228-232.

Matsuura, T. \& Sakai, H. 1996. On stochastic system representation of handwriting process and its application to signature verification. Third International Conference on Signal Processing Proceedings. pp. 1330-1333.

Mckeague, I. 2005. A statistical model for signature verification. American Statistical Association 100: 231-241.

NtZoufras, I. 2009. Bayesian Modeling Using WinBUGS. New York: John Wiley and Sons.

Plamondon, R. \& Srihari, S.N. 2000. On-line and off-line handwriting recognition: A comprehensive survey. IEEE Transactions on Pattern REcognition and Machine Intelligence 22(1): 63-84.

Ramsay, J.O. 2000. Functional components of variation in handwriting. Journal of the American Statistical Association 95: 9-15.

Ramsay, J.O.\& Silverman, B.W. 1997. Functional Data Analysis New York: Springer.

Sansone, C. \& Vento, M. 2000. Signature verification: Increasing performance by a multi-stage system. Pattern Analysis \& Applications 3: 169-181.

Srihari, S.N., Kuzhinjedathu, K., Srinivasan, H., Huang, C. \& Pu, D. 2008. Signature verification using a Bayesian approach. Lecture Notes in Computer Science 5158: 192-203.

Srihari, S.N., Xu, A. \& Kalera, M.K. 2004. Learning strategies and classification methods for off-line signature verification. Proc.of the 7th Int. Workshop on Frontiers in handwriting recognition (IWFHR). pp. 161-166.

van Lieshout, M.N.M. 2000. Markov Point Processes and their Applications. London: Imperial College Press.

\section{Behboudi*}

Department of Statistics

Science and Research Branch, Islamic Azad University, Tehran Iran

E. Pasha

Department of Mathematics and Computer Science

Kharazmi University, Karaj

Iran

\section{K. Shafie}

Department of Applied Statistics and Research Methods

College of Education \& Behavioral Sciences

University of Northern Colorado

Greeley, 80639 Colorado

USA 
*Corresponding author; email: m.behboudi@srbiau.ac.ir

Received: 5 June 2013

Accepted: 15 June 2015 